\title{
Prevalence data on all Ghent features in a cross-sectional study of 87 adults with proven Marfan syndrome
}

\author{
Svend Rand-Hendriksen ${ }^{\star 1,7}$, Rigmor Lundby ${ }^{2,7}$, Lena Tjeldhorn ${ }^{3}$, Kai Andersen ${ }^{4}$, \\ Jon Offstad ${ }^{5}$, Svein Ove Semb ${ }^{6}$, Hans-Jørgen Smith ${ }^{2,7}$, Benedicte Paus ${ }^{3}$ and Odd Geiran ${ }^{4,7}$ \\ ${ }^{1}$ TRS National Resource Centre for Rare Disorders, Sunnaas Rehabilitation Hospital, Nesoddtangen, Norway; \\ ${ }^{2}$ Department of Radiology, Rikshospitalet University Hospital, Oslo, Norway; ${ }^{3}$ Department of Medical Genetics, Ulleval \\ University Hospital, Oslo, Norway; ${ }^{4}$ Department of Thoracic and Cardiovascular Surgery, Rikshospitalet University \\ Hospital, Oslo, Norway; ${ }^{5}$ Department of Cardiology, Rikshospitalet University Hospital, Oslo, Norway; ${ }^{6}$ Department of \\ Ophthalmology, Center for Eye Research, Ullevaal University Hospital, University of Oslo, Oslo, Norway; ${ }^{7}$ Faculty \\ Division Rikshospitalet, University of Oslo, Oslo, Norway
}

The prevalence of each single feature in the Ghent criteria in patients with Marfan syndrome (MFS) is not known. To elucidate this, a cross-sectional study of 105 adults with presumed MFS was carried out. All patients were examined by the same group of investigators with standardized and complete assessment of all features in the Ghent criteria. Eighty-seven (83\%) fulfilled the criteria in 56 different variants. The most prevalent major criterion in Ghent-positive persons was dural ectasia (91\%), followed by major genetic criterion (89\%) and ectopic lenses (62\%). In 14 persons (16\%), the diagnosis was dependent on the dural findings. In all, 79\% fulfilled both major dural and major genetic (positive family history and/or FBN1 mutation) criteria, suggesting that most patients with MFS might be identified by investigating these criteria. A history or finding of ascending aortic disease was present in 46 patients (53\%). This low prevalence might partly reflect a high number of diagnosed patients encompassing the whole spectrum of the syndrome. The study confirms the need to examine for the complete set of features in the Ghent criteria to identify all patients with MFS. The majority of persons with MFS might be identified by the combined assessment of dura mater and family history, supplemented with DNA analysis in familynegative cases. The low prevalence of ascending aortic disease might indicate better future prospects in an adult population than those traditionally considered.

European Journal of Human Genetics (2009) 17, 1222-1230; doi:10.1038/ejhg.2009.30; published online 18 March 2009

Keywords: dural ectasia; FBN1; lens dislocation; Marfan syndrome; protrusio acetabuli

\section{Introduction}

Marfan syndrome (MFS) is an autosomal dominant genetic connective tissue disorder diagnosed by the Ghent criteria. ${ }^{1}$

*Correspondence: Dr S Rand-Hendriksen, TRS, National Resource Centre for Rare Disorders, Sunnaas Rehabilitation Hospital, Bjornemyrveien, Nesoddtangen N. 1450, Norway.

Tel: + 476696 9000; Fax: + 476691 2576;

E-mail: svend.rand-hendriksen@sunnaas.no

Received 6 May 2008; revised 16 December 2008; accepted 6 February 2009; published online 18 March 2009
To obtain proper counselling and care of individuals with MFS, a precise diagnosis is mandatory, prolonging life expectancy and reducing disability. ${ }^{2}$ The prevalence of MFS is disputed. Two MFS prevalence studies reports 6.81/ $100.000^{3}$ and 4.6/100.000 inhabitants, respectively, ${ }^{4}$ whereas the usually cited prevalence is $1-2 / 10.000$ inhabitants. ${ }^{5}$

The Ghent criteria describe a number of variably well-defined symptoms and signs. They are categorized as 'major criteria', 'manifestations' (in the skeletal system) and 'minor criteria', which alone or combined indicate the 
'involvement of an organ system' or the 'fulfilment of a major criterion'. Fulfilment of at least two major criteria in two different organ systems and the involvement of a third organ system are required to give the diagnosis.

Although FBN1 is the only gene included in the Ghent nosology, ${ }^{1}$ FBN1 mutations are also found in individuals not fulfilling the Ghent criteria, ${ }^{6,7}$ and mutations in TGFBR1 and TGFBR2 have recently been reported in individuals fulfilling the Ghent criteria. ${ }^{8,9}$

The resources needed and the logistic challenge in the examination of all organ systems may explain why clinical papers do not commonly present complete data for all variables. Furthermore, some may consider it appropriate to stop the investigations when data sufficient for a diagnosis of MFS have been acquired. This is illustrated by the fact that less than $30 \%$ of the individuals were investigated for dural ectasia and protrusio acetabuli in recent collective reviews on the basis of the FBN1 mutation database, presenting genotype-phenotype correlations. ${ }^{10,11}$ Moreover, various prevalences of 'major criteria' and 'organ involvement' are presented. The various results might in part be owing to the features being age dependent, ${ }^{12}$ as most studies present results for children or mixed age groups whereas reports for adults are rare. ${ }^{13,14}$

The need for sensitivity and specificity of the 'major criteria' and 'organ involvement' has been pointed out. ${ }^{1,15}$ However, neither prevalences nor sensitivity and specificity are known.

Thus, the purpose of this study was to explore the phenotype, the prevalence of each 'major criterion' and 'organ involvement' through a prospective and complete investigation of all features of the Ghent criteria in an adult cohort with a proven diagnosis of MFS.

\section{Patients and methods}

This study was approved by the regional ethics committee. In 2002, Norway had 4.5 million inhabitants, the majority being Caucasians. By October 2002, 179 individuals had reported themselves to the National Resource Centre for MFS (TRS) as having MFS; 134 of them were 18 years of age or older and all were citizens of Norway.

The participants in the Norwegian Marfan Syndrome Study were recruited either by an invitation letter sent to the 134 individuals above 18 years of age in the TRS database, who had registered as having MFS, where by 80 persons signed in for participation, or by an advertisement in the journal of the Norwegian Association for MFS asking for persons who have been diagnosed with MFS, or through invitations distributed in the Department of Thoracic and Cardiovascular Surgery, Rikshospitalet University Hospital to patients suspected for MFS, thereby recruiting an additional 19 persons, although the number of persons informed about the study is unknown. A total of 109 individuals thus gave their informed consent to participate, but one died before the study started, one was not able to attend while living abroad and two participants withdrew, one before and one during the study.

Consequently, the study population consisted of 105 individuals, 67 women (64\%) of median age 42 years (range 20-69 years) and 38 men (36\%) of median age 33 years (range 19-62 years).

Ninety of the 105 had earlier been given a diagnosis of MFS; 15 persons entered the study because of suspicion of MFS. All participants were Caucasian.

The 105 individuals represented 66 families. Forty-five individuals were the only representatives of their family, whereas 60 individuals were from 21 families.

To obtain valid results, the same group of investigators, using the same methods for all patients, carried out all examinations. All participants were assessed for all Ghent criteria. ${ }^{1}$ The methods of assessment of clinical and radiological examinations and cutoff limits are presented in Table $1 .^{16-18}$ Details of the mutation analysis have been published. ${ }^{19,20}$

As recommended by the authors of the Ghent criteria, the serum concentration of homocysteine was measured in all 105 individuals to exclude homocystinuria. ${ }^{1}$

When all data were present, the status for each individual was assessed with regard to each organ system (major criteria fulfilled and organ systems involved). Adding up, the number of major criteria fulfilled and organ systems involved was registered, and the individual was classified as fulfilling the Ghent criteria or not.

\section{Statistics}

All data were stored in a customized database (SPSS for Windows version 13). Descriptive statistics are reported as medians (ranges). Owing to the low number of individuals, results are reported as fractions and percentages.

\section{Results}

Of the 105 individuals, 87 persons from 57 families fulfilled the Ghent criteria (83\%), whereas 18 individuals (17\%) from 9 families did not. Of those individuals fulfilling the Ghent criteria, 77 had earlier been diagnosed with MFS, whereas MFS was suspected in 10 individuals. The summary and interpretation of all examinations and the prevalences of fulfilled major criteria and involved organ systems are presented in Table 2 and Figure 1.

The prevalence of the major criteria in the other four organ systems did not show significant differences in accordance to gender.

The 87 persons fulfilled the Ghent criteria through 56 different combinations of major criteria and organ involvement; the highest number of individuals having the same combination of criteria was 5 . Ten persons 
Table 1 Diagnostic criteria for Marfan syndrome (MFS) according to the Ghent nosology

Confirmation of the diagnosis requires the presence of at least two major criteria in two different organ systems and involvement of a third organ system. Family history/genetic is counted as an organ system

System

Method

Major criteria

Skeletal

Clinical investigation,

CT scan of thoracic cage;

scout view of spine,

CT scan of acetabuli

Ocular

Slit lamp investigation,

keratometry and ultrasound

\section{Cardiovascular}

Echocardiography, MRI of thoracic aorta. If MRI was not possible, CT of thoracic aorta below

than 1.05
Requires four of the eight manifestations listed

\section{Manifestations:}

Pectus carinatum

Pectus excavatum requiring surgery

Reduced upper to lower segment ratio

$<0.85^{\mathrm{a}}$ or Arm span to height ratio greater

Wrist and thumb signs

Scoliosis of $>20^{\circ}$ or spondylolisthesis

Reduced extension at the elbows $\left(<170^{\circ}\right)$

Medial displacement of the medial malleolus

causing pes planus

Protrusio acetabuli ${ }^{c}$ of any degree

Ectopia lentis $^{\mathrm{d}}$

Requires two of the following three minor criteria

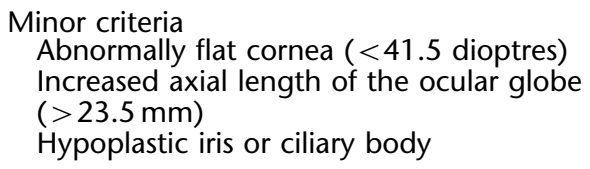

Hypoplastic iris or ciliary body

Requires the presence of at least one major criterion or one minor criterion without aortic regurgitation and involving at least the sinuses of Valsalva ${ }^{\mathrm{e}}$

Dissection of the ascending aorta

Requires two of the eight manifestations in the left column or one manifestation plus two minor criteria

Minor criteria

Highly arched palate with crowding of teeth

Facial appearance (dolicocephaly ${ }^{\mathrm{b}}$, malar hypoplasia, enophthalmos, retrognathia, downslanting palpebral fissures)

\section{Minor criteria}

Pulmonary

History, CT scan of thoracic cage

Skin and integument

History, inspection

Dura mater

MRI of lumbosacral spine.If MRI was not possible: $C T$ of lumbosacral spine
Major criterion:

Lumbosacral dural ectasia ${ }^{i}$
Criterion for involvement

Mitral valve prolapse with or without mitral valve regurgitation

Dilatation of the main pulmonary artery in the absence of valvular or peripheral pulmonic stenosis or any other obvious cause below the age of 40 years

Calcification of the mitral annulus before the age of 40 years

Dilatation or dissection of the descending thoracic or abdominal aorta below the age of 50 years

Requires at least one minor criterion

Minor criteria

Spontaneous pneumothorax

Apical blebs ${ }^{9}$

Requires at least one minor criterion

Minor criteria

Striae athrophicae (stretch marks) not associated with marked weight changes, pregnancy or repetitive stress ${ }^{h}$

Recurrent or incisional herniae

None 
Table 1 (Continued)

Confirmation of the diagnosis requires the presence of at least two major criteria in two different organ systems and involvement of a third organ system. Family history/genetic is counted as an organ system

System

Method

Major criteria

Criterion for involvement

Family history/genetic

Having a parent, child or sib who meets

None

Sequencing of coding sequences of FBN1

these diagnostic criteria independently ${ }^{j}$

Presence of a mutation in FBN1 known to

cause the Marfan syndrome ${ }^{\mathrm{k}}$

Presence of a FBN1 haplotype around FBN1,

inherited by descent, known to be associated

with unequivocally diagnosed Marfan

syndrome in the family

Our methods of investigation are indicated in italics in the first column. Where no limit for normality is given in the Ghent criteria, our chosen values are presented, where possible with references.

Our chosen limits are as follows:

a (see reference Roman et al). ${ }^{18}$

${ }^{\mathrm{b}}$ Cephal index $<0.76$ (Hall et al., 1995). ${ }^{16}$

'On the CT monitor, an ellipse is adapted aligning the inner border of the pelvis over the acetabulum. We defined protrusio acetabuli to be present when the bottom of the acetabulum protrudes into the ellipse.

${ }^{d}$ Ectopia lentis was noted when (1) the lenses had been removed due to luxation, (2) luxation; the centre of the lens is displaced from the centre of the pupil, (3) subluxation; horizontal tilt of the lens, usually the caudal part of the lens tilted posteriorly leaving space between the lens and the iris.

ePlotting body surface area (BSA) versus echocardiographic aortic diameter in sinus valsalva into age relevant normograms (from Roman et al ${ }^{17}$ ), the result lies higher than the normal upper border or graft due to dilated/dissected ascending aorta.

fPlotting BSA versus MRI or CT measured diameter of pulmonary artery into age relevant aorta normograms (from Roman et $\mathrm{ll}^{17}$ ), the result lies higher than the normal upper border.

${ }^{9}$ Blebs in the lungs on CT.

${ }^{\mathrm{h}} \mathrm{Striae}$ from puberty/before pregnancy.

'Visualization by CT or MRI of anterior meningocele (ie: dura covered spinal fluid inside the pelvic cavity); dural sac diameter S1 or distally larger than dural sac diameter L4; one or more herniations of dura along lumbosacral nerve roots; dural sac ratio $L 5>0.48$ or dural sac ratio $51>0.57$ (from Oosterhof $e t a l^{34}$ ).

'On the basis of the probands knowledge about their relatives: Lens (sub)luxation and/or operation, dilatation/dissection or operation of ascending aorta and documented FBN1 mutations.

${ }^{\mathrm{k}} \mathrm{A}$ mutation in FBN1 resulting in amino acid shift and not reported to represent a genetic polymorphism, or a large duplication/deletion in FBN1.

fulfilled all five possible major criteria, three of them also having lungs and skin and integument involved.

Of the 87 fulfilling the diagnostic criteria, 76 fulfilled the Ghent criteria on the basis of the individual examinations alone, without considering family history or mutation analyses.

Another nine individuals did fulfil one major organ criterion and had a first-degree relative independently fulfilling the Ghent criteria (positive family history) as the second major criterion and a third organ system involved.

Two individuals, both being the first in their family to be diagnosed with MFS, fulfilled one major organ criterion, had an FBN1 mutation as their second major criterion and had a third organ system involved. These two persons were the only ones to be dependent on mutation analysis to be diagnosed with MFS.

Of the 87 persons fulfilling the Ghent criteria, 14 were dependent on the dural criterion for the diagnosis.

Of the 87 individuals fulfilling the diagnostic criteria, an FBN1 mutation was found in 73 individuals, representing 44 families. $^{19,20}$ No FBN1 mutations were found in individuals not fulfilling the Ghent criteria.
Of the 18 individuals not fulfilling the diagnostic criteria, 13 had been diagnosed with MFS earlier, including a person with a highly elevated serum concentration of homocysteine $(320 \mu \mathrm{mol} / \mathrm{l})$, one person in whom a mutation in the Collagen 3 gene (COL3A1) was found and five persons from one family in whom a TGFBR2 mutation was found.

The prevalence of the features in the Ghent criteria among the 87 individuals fulfilling the diagnostic criteria in our study is presented in Table 3 for comparison with prevalences from other studies reporting the prevalence of two or more major criteria. ${ }^{11,21-29}$

The prevalence of having at least two major criteria fulfilled in our study is presented in Figure 2.

\section{Discussion}

This study confirms the need for the complete Ghent criteria to identify all patients with MFS, although the majority of them might be identified by combined assessment of dura and family history, supplemented with DNA analysis in family-negative cases. 
Table 2 Prevalence of the individual features.

\begin{tabular}{|c|c|c|c|}
\hline & $\begin{array}{l}\text { Total, } \\
\mathrm{N}=105\end{array}$ & $\begin{array}{l}\text { Fulfilling } \\
\text { Ghent, } \\
\mathrm{N}=87\end{array}$ & $\begin{array}{l}\text { Not Ghent, } \\
\mathrm{N}=18\end{array}$ \\
\hline $\begin{array}{l}\text { Pectus carinatum } \\
\text { Pectus excavatum requiring surgery } \\
\text { Reduced upper to lower segment ratio }(<0.85) \text { or arm span to height ratio greater than } 1.05 \\
\text { Wrist and thumb signs } \\
\text { Scoliosis of }>20^{\circ} \text { or spondylolisthesis } \\
\text { Reduced extension at the elbows }\left(<170^{\circ}\right) \\
\text { Medial displacement of the medial malleolus causing pes planus } \\
\text { Protrusio acetabuli of any degree } \\
\text { Fulfilling skeletal major } \\
\text { Skeletal system involved }\end{array}$ & $\begin{array}{r}60 \\
6 \\
48 \\
21 \\
24 \\
29 \\
37 \\
56 \\
34 \\
90\end{array}$ & $\begin{array}{r}57 \\
6 \\
43 \\
20 \\
23 \\
26 \\
32 \\
52 \\
33 \\
82\end{array}$ & $\begin{array}{l}3 \\
- \\
5 \\
1 \\
1 \\
3 \\
5 \\
4 \\
1 \\
8\end{array}$ \\
\hline $\begin{array}{l}\text { Abnormally flat cornea ( }<41.5 \text { dioptres) } \\
\text { Increased axial length of the ocular globe }(>23.5 \mathrm{~mm} \text { ) } \\
\text { Hypoplastic iris or ciliary body (transillumination of the iris, loss of iris crypts) } \\
\text { Fulfilling ocular major= ectopia lentis } \\
\text { Ocular system involved }\end{array}$ & $\begin{array}{r}47 \\
71 \\
3 \\
56 \\
37\end{array}$ & $\begin{array}{r}46 \\
62 \\
3 \\
54 \\
36\end{array}$ & $\begin{array}{l}1 \\
9 \\
- \\
2 \\
1\end{array}$ \\
\hline $\begin{array}{l}\text { Mitral valve prolaps with or without mitral valve regurgitation } \\
\text { Dilatation of the main pulmonary artery below the age of } 40 \text { years, see reference Roman et al }{ }^{17} \\
\text { Calcification of the mitral annulus below the age of } 40 \text { years } \\
\text { Dissection of the descending thoracic or abdominal aorta below the age of } 50 \text { years (B-dissection) } \\
\text { Dilatation of the descending thoracic or abdominal aorta below the age of } 50 \text { years } \\
\text { Fulfilling cardiovascular major = dilatation or dissection of ascending aorta } \\
\text { Cardiovascular system involved } \\
\text { A-dissection before the study } \\
\text { Graft in ascending aorta before the study }\end{array}$ & $\begin{array}{r}14 \\
- \\
1 \\
5 \\
- \\
51 \\
57 \\
5 \\
31\end{array}$ & $\begin{array}{r}12 \\
- \\
1 \\
5 \\
- \\
46 \\
51 \\
5 \\
30\end{array}$ & $\begin{array}{c}2 \\
- \\
- \\
- \\
- \\
5 \\
6 \\
- \\
1\end{array}$ \\
\hline $\begin{array}{l}\text { Apical blebs } \\
\text { Spontaneous pneumothorax } \\
\text { Lungs involved }\end{array}$ & $\begin{array}{r}22 \\
6 \\
24\end{array}$ & $\begin{array}{r}16 \\
5 \\
18\end{array}$ & $\begin{array}{l}6 \\
1 \\
6\end{array}$ \\
\hline $\begin{array}{l}\text { Recurrent or incisional herniae } \\
\text { Striae atrophicae (stretch marks) from puberty } \\
\text { Skin and integument involved }\end{array}$ & $\begin{array}{l}10 \\
70 \\
74\end{array}$ & $\begin{array}{r}8 \\
60 \\
63\end{array}$ & $\begin{array}{r}2 \\
10 \\
11\end{array}$ \\
\hline $\begin{array}{l}\text { Dural sac diameter S1 or distal }>\text { dural sac diameter } L 4 \\
\text { DSR L5 }>0.48 \text { and/or DSR S1 >0.57 } \\
\text { One or more herniations of dura along lumbosacral nerve roots } \\
\text { Anterior meningocele } \\
\text { Fulfilling dural major }\end{array}$ & $\begin{array}{l}43 \\
65 \\
65 \\
29 \\
82\end{array}$ & $\begin{array}{l}42 \\
64 \\
63 \\
29 \\
79\end{array}$ & $\begin{array}{l}1 \\
1 \\
2 \\
- \\
3\end{array}$ \\
\hline $\begin{array}{l}\text { Having a parent, child or sibling who meets these diagnostic criteria independently } \\
\text { The presence of a mutation in FBN1 known to cause the Marfan syndrome } \\
\text { Fulfilling genetic major }\end{array}$ & $\begin{array}{l}58 \\
73 \\
79\end{array}$ & $\begin{array}{l}56 \\
73 \\
77\end{array}$ & $\begin{array}{c}2 \\
\frac{2}{2}\end{array}$ \\
\hline
\end{tabular}

$N=105$. Major criteria and affected organ systems are indicated in italics.

Using the full version of the Ghent criteria on 105 individuals refuted the MFS diagnosis in 13 of 90 patients who had been diagnosed earlier, and verified the diagnosis in 10 of 15 persons with suspected MFS. The 56 different ways of fulfilling the Ghent criteria in this cohort illustrate the need for the complete set of criteria and the problems with differential diagnosis versus other genetic connective tissue disorders. Our cohort represents persons with given or suspected diagnosis of MFS; the primary clinical result of our investigations is the separation of individuals fulfilling the Ghent criteria from individuals suspected for MFS, not fulfilling the Ghent criteria.
The Ghent criteria require at least two major criteria to be fulfilled. The major criteria in five organ systems have equal value and validity in the diagnostic process. Even so, the concept of severity of Marfan phenotypes has been presented. ${ }^{30-32}$ 'Severe Marfan syndrome' often seems to be understood as 'severe cardiovascular pathology', irrespective of age, not as a person fulfilling many major criteria. The term 'severity' in accordance with the MFS has yet to be defined.

In spite of the great diversity of combinations, a combination of the dural major criterion and the presence of genetic major (positive family history and/or mutation 


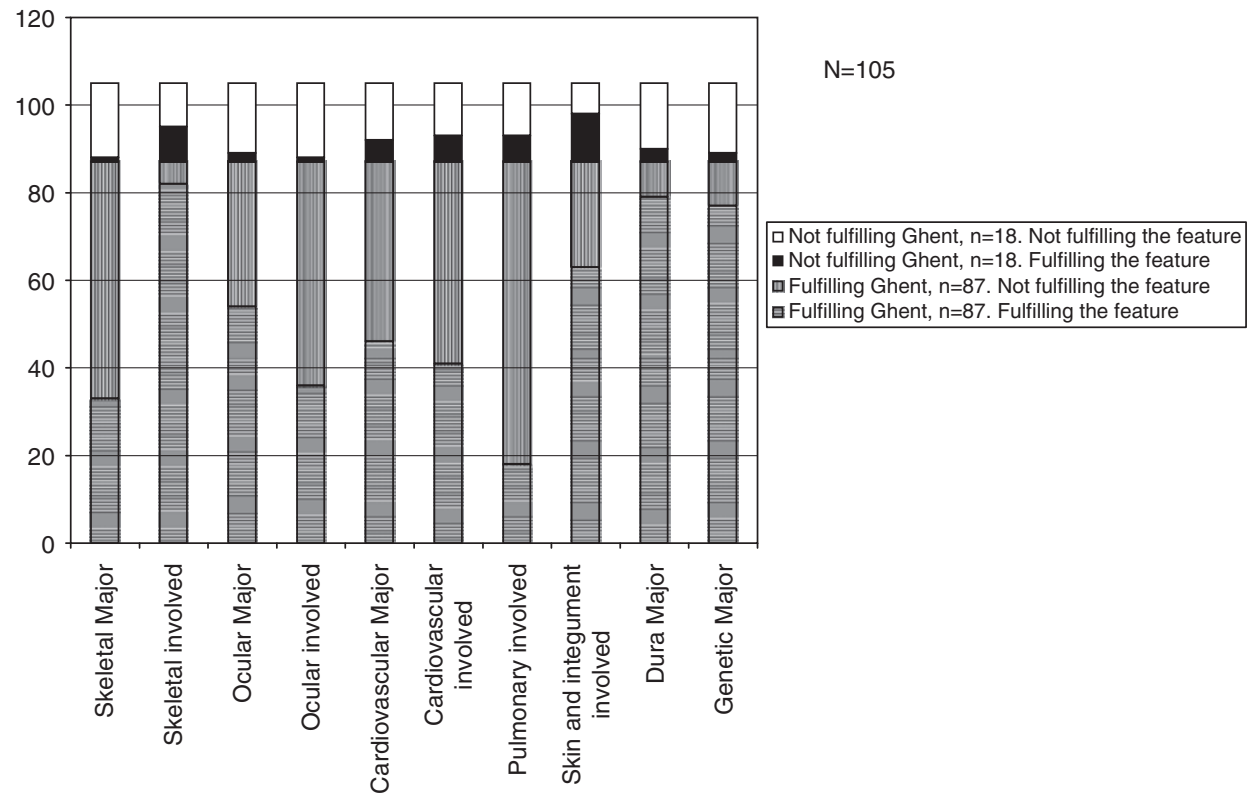

Figure 1 Number of persons fulfilling major criteria and having organ systems involved. Over all, 105 persons with given or suspected diagnosis of Marfan syndrome. Order as in the Ghent paper.

in FBN1) criteria could identify 69 out of 87 (79\%) affected individuals with MFS in our cohort.

The lack of investigations on the dura in many other studies and the problems resulting from this have been pointed out by Adès et al $\mathrm{l}^{33}$

Dural ectasia was the most frequent major criterion present in individuals fulfilling the Ghent criteria (91\%) comparable with the prevalences found in other studies. ${ }^{15,21,34}$ It is noteworthy that $16 \%$ of the individuals fulfilling Ghent were dependent on this major criterion. If dura had not been investigated, they would not have been given the diagnosis.

In our study, a positive family history (64\%) was found less often than expected. This is probably because of the high number of probands in our study and because investigations of family members were not carried out for relatives who had not signed in for the study; the participants were asked about their relatives having obvious major manifestations as known aortic dilatation or operation, known ectopic lenses or lenses removed and known mutations; see subtext to Table $1 .^{10}$

In spite of sequencing all coding parts of FBN1 and searching for large deletions and duplications, the presence of FBN1 mutations (44 probands out of 57 probands fulfilling the Gent criteria, $77 \%$ ), is lower in our study than in that commonly reported among individuals fulfilling the Ghent criteria. ${ }^{2,35}$ All patients with a mutation in FBN1 did fulfil the Ghent criteria; thus, no other 'type-1 fibrillinopathies' were found. ${ }^{7}$ Investigating dura in all cases may be the reason for this discrepancy.
The prevalence of ascending aortic disease among individuals fulfilling the Ghent criteria in our study (53\%) (Table 2), more often found in men than in women, is among the lowest reported. We have not found papers reporting different prevalences of major aortic pathology depending on gender. However, families have been observed in which the men are more likely to have earlier onset aortic enlargement, more rapid enlargement and earlier dissection than the women in the family. ${ }^{36}$ One might speculate whether our results represent a true difference between genders or differences between recruiting men and women. In our clinical work, it seems that a man needs a serious organ affection as a trigger for searching for help in their coping process. The prevalence of mitral valve prolapse (MVP) was also low compared with that in other studies, which, however, presents cohorts of children or mixed age groups. ${ }^{37-39,29}$ We did not encounter any patient with a dilated pulmonary trunk who was below the age of 40 years, using the aortic normograms as suggested in the Ghent paper. Using Nollen's upper limit of normality for the pulmonary trunk, $34.8 \mathrm{~mm}, 13$ out of 87 persons fulfilling Ghent had enlarged pulmonary trunk (median diameter $30 \mathrm{~mm}$; range $23-38 \mathrm{~mm}$ ). ${ }^{40}$

As most studies that were found have emerged from specialized 4th level cardiovascular centres serving individuals with severe aortic or cardiovascular disease, patient selection may explain the high prevalence of cardiovascular pathology in other reports.

The acronym 'MASS phenotype' has been suggested to emphasize the involvement of the mitral valve, aorta, 
Table 3 Prevalence (\%) of major criteria and affected organ systems in the Ghent criteria in papers presenting $\geqslant 2$ organ systems

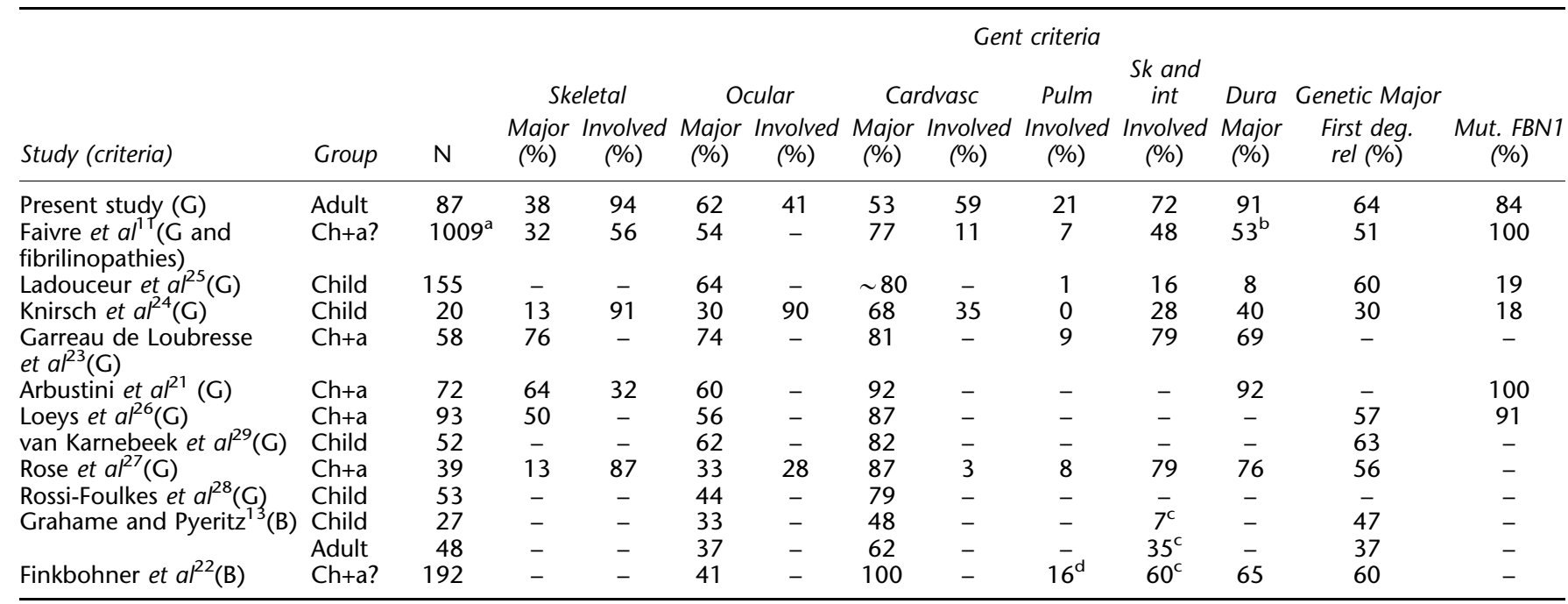

G, Ghent criteria; B, Berlin criteria; Sk and int, skin and integument; first deg. rel., first-degree relative independently fulfilling the Ghent criteria; Mut., mutation.

${ }^{a}$ Information about varying number of persons for different features.

b154 persons out of 292 persons where dura mater was investigated.

conly Striae athrophica.

${ }^{\mathrm{d}}$ Spontaneous pneumothorax.

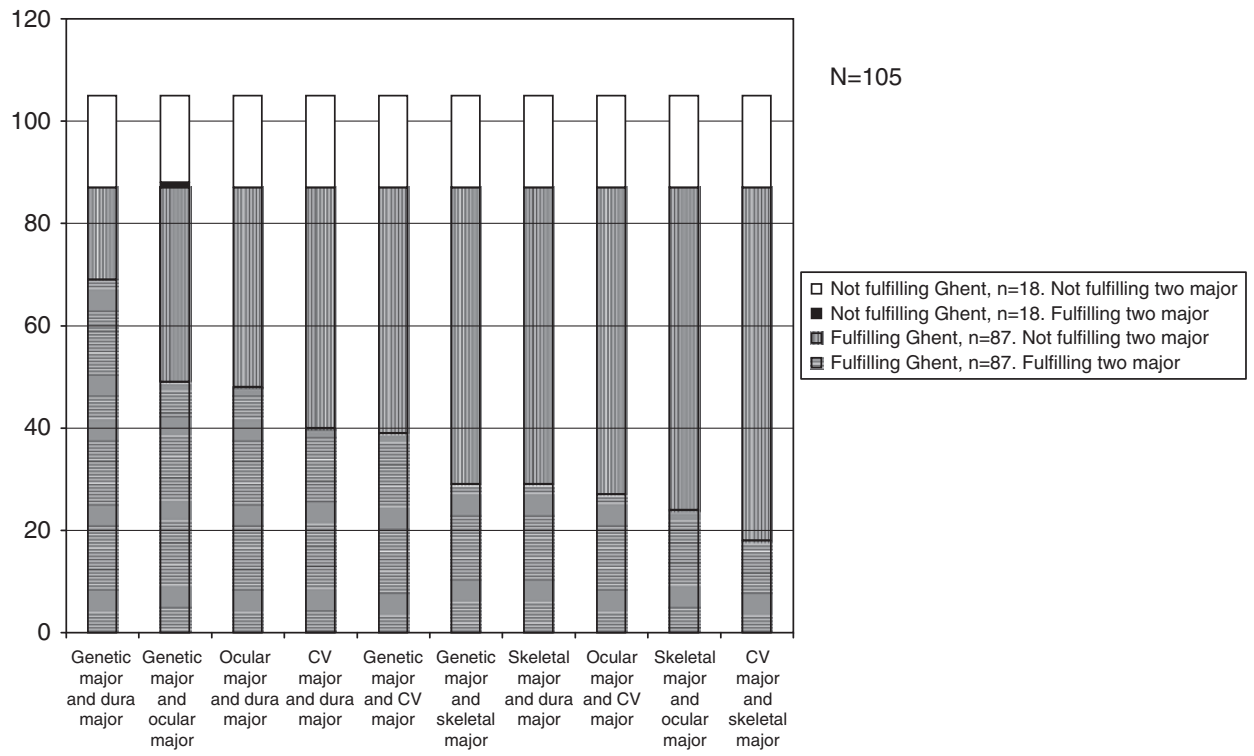

Figure 2 Number of persons fulfilling two major criteria, organized after prevalence. $N=105$.

skeleton and skin in persons who could not be classified in accordance to the Berlin nosology. ${ }^{41}$ In our study, no patient fitted into the 'MASS phenotype.'

Our cohort represents persons recruited through all medical specialities and through the patient organization.
In addition, the social security system in Norway does prevent economical constraints for diagnosis and participation in the study. Our study cohort may therefore be more representative for an adult population with MFS of different phenotypes. 
Most studies of cardiovascular pathology in MFS have been carried out on groups with low mean age; therefore the natural history of the aging Marfan aorta is not well known. In our adult cohort, we expect the features of MFS, including cardiovascular manifestations, to be present, and it is unlikely that typical features would disappear during life. Although a further development of aortic pathology in our cohort in the next few decades cannot be excluded, our study indicates that dilatation and dissection of the ascending aorta and of MVP may be found more seldom in adults than in cohorts including Marfan children and adolescents. This underlines the more severe consequences of manifest cardiovascular disorders in the lower age groups, sometimes resulting in early cardiovascular death, as illustrated by Gray et al. ${ }^{42}$ Thus, a patient selection on the basis of the natural history of the disease may have taken place. However, if the low prevalence of cardiovascular pathology is representative for adult persons with MFS, it will influence the overall clinical outcome and, consequently, the estimated individual prognosis when counselling adult persons with MFS.

The prevalence of lens subluxation or luxation in our study, $62 \%$, is the average of what is reported, ${ }^{43,4}$ whereas the prevalence of fulfilling the major criteria for the skeletal system was 38\%; other reports show large variation. ${ }^{23,24}$ The involvement of skin and integument is comparable with the highest prevalence published. ${ }^{23,27,44}$

Spontaneous pneumothorax has been rare in our cohort, whereas blebs in the lungs were found more often. $^{45}$ This can partially be explained by different imaging techniques.

The patient with two major criteria, but not fulfilling the Ghent criteria, had subluxation of one lens and a mother independently fulfilling the Ghent criteria. The mother had the family mutation, whereas the patient did not.

Family history, aortic disease, loose lenses or Marfanoid habitus usually raises the suspicion of MFS. Our results indicate that at the time of suspecting the MFS in an adult, an early investigation using MRI of the lumbosacral spine to detect dural ectasia is appropriate. When MFS is confirmed, the individual should be referred to a interdisciplinary 'Marfan centre' for individual counselling, follow-up and care. $^{2}$ Even if close to $80 \%$ of our cohort could have settled the diagnosis by dura and family/genetic major criteria, a complete examination of all organ systems in the Ghent criteria should be carried out.

The broad variety of ways of fulfilling the Ghent criteria illustrates the need for individual counselling on the basis of the individuals' own signs and findings in all organ systems.

Longitudinal studies of groups of persons fulfilling the Ghent criteria might indicate whether some variants may have more benign natural history than do others. Until then, all persons fulfilling the Ghent criteria should have ophthalmological and aortic controls for the rest of their lives.

A strength of our study is the defined population from which the participants are recruited (the Norwegian population of 4.5 million inhabitants) and that the same group of investigators using the same methods carried out all the examinations with close to no missing data. The complete investigation in all cases may have increased the number of individuals fulfilling the Ghent criteria through combinations of features not depending on the cardiovascular system. The relatively small size of our cohort might possibly influence our results. However, most commonly, current papers provide incomplete clinical information as compared with the extensive and complete investigations performed in this study.

A limitation is that our study population was skewed for gender, women being in surplus. This reflects the representation of gender among individuals over 18 years of age with MFS, who have registered themselves at the National Resource Centre for Rare Disorders, TRS.

In conclusion, using the complete list of features in the Ghent criteria, out of 105 adult individuals with given or suspected diagnosis of MFS, 87 fulfilled the Ghent criteria in 56 different variants. The large number of variants shows the need for using the complete set of features in the Ghent criteria. Dural ectasia is the major criterion most often present in persons fulfilling the Ghent criteria (91\%), followed by FBN1 mutations (84\%), positive family history (64\%) and ectopic lenses (62\%). Dilatation or dissection of the ascending aorta was only found in 53\% of Ghent-positive persons (30 of them operated), one of the lowest prevalences published. The gender difference observed in the prevalence of major aortic pathology may be real or may represent a selection bias. Having dural ectasia as well as fulfilling the major genetic criterion (positive family history and/or FBN1 mutation) was the combination of fulfilling two major criteria most often found, with $79 \%$ of persons fulfilling the Ghent criteria, indicating an early investigation of those systems when suspicion of MFS has been raised in adults. The low prevalence of cardiovascular pathology might indicate better future prospects in an adult population than those traditionally considered. More studies are needed describing the prevalence of all Ghent features, thereby making it possible to calculate the sensitivity and specificity for each feature.

\footnotetext{
Acknowledgements

We thank Finn Lilleås, MD and Ina Ghisolfi, technologist, Diakonhjemmet Hospital, Oslo, Norway for performing the radiological investigations. This study has been funded by (South-) Eastern Norway Regional Health Authority (Helse (Sør-)Øst RHF); TRS, a National Resource Centre for Rare Disorders; the Stokbaks Heart Foundation and the Kirkevoll Memory Foundation.
} 


\section{References}

1 De Paepe A, Devereux RB, Dietz HC, Hennekam RC, Pyeritz RE: Revised diagnostic criteria for the Marfan syndrome. Am J Med Genet 1996; 62: 417-42.

2 Ammash NM, Sundt TM, Connolly HM: Marfan syndromediagnosis and management. Curr Probl Cardiol 2008; 33: 7-39.

3 Gray JR, Bridges AB, Faed MJ et al: Ascertainment and severity of Marfan syndrome in a Scottish population. J Med Genet 1994; 31: $51-54$.

4 Fuchs J: Marfan syndrome and other systemic disorders with congenital ectopia lentis. A Danish national survey. Acta Paediatr 1997; 86: 947-952.

5 Pyeritz RE: The Marfan syndrome. Annu Rev Med 2000; 51: 481-510.

6 Collod-Beroud G, Boileau C: Marfan syndrome in the third Millennium. Eur J Hum Genet 2002; 10: 673-681.

7 Collod-Beroud G, Le Bourdelles S, Ades L et al: Update of the UMD-FBN1 mutation database and creation of an FBN1 polymorphism database. Hum Mutat 2003; 22: 199-208.

8 Disabella E, Grasso M, Marziliano N et al: Two novel and one known mutation of the TGFBR2 gene in Marfan syndrome not associated with FBN1 gene defects. Eur J Hum Genet 2006; 14: 34-38.

9 Singh KK, Rommel K, Mishra A et al: TGFBR1 and TGFBR2 mutations in patients with features of Marfan syndrome and Loeys-Dietz syndrome. Hum Mutat 2006; 27: 770-777.

10 Faivre L, Collod-Beroud G, Loeys BL et al: Effect of mutation type and location on clinical outcome in 1,013 probands with Marfan syndrome or related phenotypes and FBN1 mutations: an International Study. Am J Hum Genet 2007; 81: 454-466.

11 Faivre L, Collod-Beroud G, Child A et al: Contribution of molecular analyses in diagnosing Marfan syndrome and type I fibrillinopathies: an International Study of 1009 Probands. J Med Genet 2008; 45: 384-390.

12 Dean JC: Marfan syndrome: clinical diagnosis and management. Eur J Hum Genet 2007; 15: 724-733.

13 Grahame R, Pyeritz RE: The Marfan syndrome: joint and skin manifestations are prevalent and correlated. Br J Rheumatol 1995; 34: $126-131$.

14 Hasan A, Poloniecki J, Child A: Ageing in Marfan syndrome. Int J Clin Pract 2007; 61: $1308-1320$.

15 Fattori R, Nienaber CA, Descovich B et al: Importance of dural ectasia in phenotypic assessment of Marfan's syndrome. Lancet 1999; 354: 910-913.

16 Hall JG, Froster-Iskenius UG, Allanson JE: Handbook of normal physical measurements, Oxford; New York; Toronto, Oxford University Press. ISBN 019 261696, 1995; vol, pp 270-275.

17 Roman MJ, Devereux RB, Kramer-Fox R, O'Loughlin J: Twodimensional echocardiographic aortic root dimensions in normal children and adults. Am J Cardiol 1989; 64: 507-512.

18 Roman MJ, Devereux RB, Kramer-Fox R, Spitzer MC: Comparison of cardiovascular and skeletal features of primary mitral valve prolapse and Marfan syndrome. Am J Cardiol 1989; 63: 317-321.

19 Rand-Hendriksen S, Tjeldhorn L, Lundby R et al: Search for correlations between FBN1 genotype and complete Ghent phenotype in 44 unrelated Norwegian patients with Marfan syndrome. Am J Med Genet A 2007; 143: 1968-1977.

20 Tjeldhorn L, Rand-Hendriksen S, Gervin K et al: Rapid and efficient FBN1 mutation detection using automated sample preparation and direct sequencing as the primary strategy. Genet Test 2006; 10: 258-264.

21 Arbustini E, Grasso M, Ansaldi S et al: Identification of sixty-two novel and twelve known FBN1 mutations in eighty-one unrelated probands with Marfan syndrome and other fibrillinopathies. Hum Mutat 2005; 26: 494.

22 Finkbohner R, Johnston D, Crawford ES, Coselli J, Milewicz DM: Marfan syndrome. Long-term survival and complications after aortic aneurysm repair. Circulation 1995; 91: 728-733.
23 Garreau de Loubresse C, Mullins MM, Moura B et al: Spinal and pelvic parameters in Marfan's syndrome and their relevance to surgical planning. J Bone Joint Surg Br 2006; 88: 515-519.

24 Knirsch W, Kurtz C, Haffner N et al: Dural ectasia in children with Marfan syndrome: a prospective, multicenter, patient-control study. Am J Med Genet A 2006; 140: 775-781.

25 Ladouceur M, Fermanian C, Lupoglazoff JM et al: Effect of betablockade on ascending aortic dilatation in children with the Marfan syndrome. Am J Cardiol 2007; 99: 406-409.

26 Loeys B, De Backer J, Van Acker P et al: Comprehensive molecular screening of the FBN1 gene favors locus homogeneity of classical Marfan syndrome. Hum Mutat 2004; 24: 140-146.

27 Rose PS, Levy HP, Ahn NU et al: A comparison of the Berlin and Ghent nosologies and the influence of dural ectasia in the diagnosis of Marfan syndrome. Genet Med 2000; 2: 278-282.

28 Rossi-Foulkes R, Roman MJ, Rosen SE et al: Phenotypic features and impact of beta blocker or calcium antagonist therapy on aortic lumen size in the Marfan syndrome. Am J Cardiol 1999; 83: $1364-1368$

29 van Karnebeek CD, Naeff MS, Mulder BJ, Hennekam RC, Offringa M: Natural history of cardiovascular manifestations in Marfan syndrome. Arch Dis Child 2001; 84: 129-137.

30 Gray JR, Davies SJ: A clinical severity grading scale for Marfan syndrome. J Med Genet 1996; 33: 758-759.

31 Pepe G, Lapini I, Evangelisti L et al: Is ectopia lentis in some cases a mild phenotypic expression of Marfan syndrome? Need for a long-term follow-up. Mol Vis 2007; 13: 2242-2247.

32 Putnam EA, Cho M, Zinn AB et al: Delineation of the Marfan phenotype associated with mutations in exons $23-32$ of the FBN1 gene. Am J Med Genet 1996; 62: 233-242.

33 Ades LC, Holman KJ, Brett MS, Edwards MJ, Bennetts B: Ectopia lentis phenotypes and the FBN1 gene. Am I Med Genet 2004; 126A: $284-289$.

34 Oosterhof T, Groenink M, Hulsmans FJ et al: Quantitative assessment of dural ectasia as a marker for Marfan syndrome. Radiology 2001; 220: 514-518.

35 Mizuguchi T, Matsumoto N: Recent progress in genetics of Marfan syndrome and Marfan-associated disorders. J Hum Genet 2007; 52: 1-12.

36 Byers PH: Marfan families have been observed in which men are more likely to have earlier onset aortic enlargement, more rapid enlargement and earlier dissection than the women in the family. Personal communication 2008.

37 Chan KL, Callahan JA, Seward JB, Tajik AJ, Gordon H: Marfan syndrome diagnosed in patients 32 years of age or older. Mayo Clin Proc 1987; 62: 589-594.

38 Das BB, Taylor AL, Yetman AT: Left ventricular diastolic dysfunction in children and young adults with Marfan syndrome. Pediatr Cardiol 2006; 27: 256-258.

39 De Backer J, Loeys B, Devos D et al: A critical analysis of minor cardiovascular criteria in the diagnostic evaluation of patients with Marfan syndrome. Genet Med 2006; 8: 401-408.

40 Nollen GJ, van Schijndel KE, Timmermans J et al: Pulmonary artery root dilatation in Marfan syndrome: quantitative assessment of an unknown criterion. Heart 2002; 87: 470-471.

41 Glesby MJ, Pyeritz RE: Association of mitral valve prolapse and systemic abnormalities of connective tissue. A phenotypic continuum. JAMA 1989; 262: 523-528.

42 Gray JR, Bridges AB, West RR et al: Life expectancy in British Marfan syndrome populations. Clin Genet 1998; 54: 124-128.

43 Maumenee IH: The eye in the Marfan syndrome. Trans Am Ophthalmol Soc 1981; 79: 684-733.

44 Cohen PR, Schneiderman P: Clinical manifestations of the Marfan syndrome. Int J Dermatol 1989; 28: 291-299.

45 Wood JR, Bellamy D, Child AH, Citron KM: Pulmonary disease in patients with Marfan syndrome. Thorax 1984; 39: 780-784. 